\title{
REFORMA E INNOVACIÓN EDUCATIVA CONSIDERACIONES TEÓRICAS PARA LA INVESTIGACIÓN CRÍTICA
}

\author{
Guillermo Miranda Camacho ${ }^{1}$
}

\section{Resumen}

Este ensayo es un acercamiento crítico a la reforma e innovación educativas. Nuestro interés es examinar algunas dimensiones de la naturaleza, fundamentación ideológico-política, y características comparativas entre estas dos manifestaciones del cambio educativo. Para acometer esta tarea, iniciamos la exposición con un análisis de los principales hechos que configuran el marco sociohistórico de la irrupción de las reformas e innovaciones educativas. En segundo lugar, exploramos algunas características fundamentales, en perspectiva comparada, de la reforma e innovación educativas. En la tercera sección, exponemos algunos elementos que consideramos básicos para un acercamiento hermenéutico crítico de modelos teóricos de las reformas educativas. En un cuarto momento, nos adentramos en el conocimiento de la dimensión histórico-estructural de las reformas educativas, como procesos que tienen lugar en un proceso de cambio del sistema social. En la quinta y última parte, realizamos un excurso teórico respecto de las funciones educativas como referentes socioestructurales de las reformas e innovaciones educativas. Este trabajo es parte de un proceso, que hemos sistematizado en otros ensayos, en el interés de aportar elementos para una visión hermenéutica crítica de los procesos de cambio educativo, y constituye un producto de la Cátedra "Educación, Desarrollo y Democracia: Uladislao Gámez Solano", del CIDE.

Palabras claves: reforma educativa, innovación educativa, cambio educativo, crisis de legitimación, estrategia de legitimación, hermenéutica crítica

\section{Abstract}

In this essay we propose to do a critical approach to the education reform and innovation. Our interest is to examine the

1 Sociólogo y Doctor en Ciencias de la Educación (Universidad de Málaga, España). Académico del Centro de Investigación y Docencia en Educación (CIDE) de la Universidad Nacional. Coordinador de la Cátedra "Educación, Democracia y Desarrollo: Uladislao Gámez Solano", del CIDE. 
nature, ideological - political fundament and comparative characteristics between two manifestations of the educational change. To accomplish this task we initiate our exposition with an analysis of the principal sociohistoric milestones witch configured the sociohistoric framework of the beginning of the education changes and innovations: the technological boom of the eighties decade. In second place, we explore some fundamental characteristics, in a comparative perspective, of the education changes and innovations. In a third moment, we expos, in a succinct way, some elements that we considerate basics to a hermeneutical and critical approach, to the models in theory of the educational changes. In the forth section, we move deeper in to the knowledge of the historical - structural dimension of the educational changes, as process witch take place in the changes of the social system. In the fifth and last part we do a theory excursus about the educational functions as sociostructural references to the education changes and innovations. This work is part of a process, that we systematize in other essays, with the intentions of contribute with elements to a critical hermeneutical vision of the process of educational changes.

Keywords: educational reform, educational innovation, teaching change, crisis of legitimacy, legitimacy strategy, critical hermeneutical.

\section{Marco sociohistórico de las reformas e innovaciones educativas}

$F^{n}$ el curso de la década de los ochenta, el cambio y las mejoras educativas se intensificaron en un periodo de cambios estructurales acelerados promovidos para enfrentar la crisis económica mundial que se había iniciado en 1973. En este contexto, las innovaciones tecnológicas aplicadas a los procesos de producción alcanzan un gran desarrollo, y se manifiestan como un factor decisivo en el desarrollo de los cambios estructurales, necesarios para el relanzamiento del proceso de acumulación ampliada de capital. Esto puso de manifiesto el hecho de que las crisis se toman en el punto de inflexión de las "oleadas de innovación tecnológica", que caracterizan los ciclos acumulativos. Concluida la crisis se reinicia la expansión acumulativa de capital incorporando las innovaciones tecnológicas de manera masiva al proceso productivo: las tecnologías de punta que en la fase acumulativa anterior a la crisis se venían desarrollando y las que han surgido en la experiencia compulsiva de la propia crisis (Moral y Raymond, 1986). Esto aconteció con la crisis de acumulación de capital, que se inició a principios de los setenta, y, como respuesta, tuvo lugar el boom de las innovaciones tecnológicas que se manifestó desde la década de 1980. 
Surge en este contexto una marcada tendencia a la diversificación de la investigación y de las aplicaciones tecnológicas que favorece la productividad económica y, por lo tanto, el crecimiento de la producción industrial masiva. El desarrollo de la tecnología alcanza una dimensión de primer orden y su vertiginosa expansión inicia un proceso característico de la modernidad tardía.

El cambio y las mejoras de las escuelas, y un decidido afán por la renovación de la enseñanza, adquieren una particular importancia que se pone de manifiesto en la década de los ochenta. La expansión de los cambios que se manifiestan en todos los órdenes del sistema social impone al sistema educativo respuestas a este fenómeno creciente en ese periodo, con marcadas tendencias de crecimiento en las décadas venideras. La importancia atribuida al cambio tecnológico "revaloriza" el concepto de innovación en la intención generalizada por explotar al máximo la aplicación del conocimiento científico a casi la totalidad de las esferas de la actividad humana.

Es difícil pensar en alguna dimensión de la vida cotidiana en el mundo occidental en que las personas no tengan contacto o reciban el influjo de la tecnología y el espíritu innovador que le acompaña en su significado práctico. La humanidad de hoy vive, ciertamente, envuelta y condicionada por el influjo tecnológico que determina todas las esferas de su vida. En el mundo subdesarrollado y dependiente, la presencia o el impacto de la revolución tecnológica está presente en algún grado, como un factor que profundiza y condiciona la dependencia de los grandes centros hegemónicos capitalistas.

La vida social ha experimentado un sensible cambio en casi la totalidad de sus ámbitos, sino en todos, por la influencia del desarrollo tecnológico, lo mismo ha sucedido en el "mundo de la vida"2 que intersubjetivamente comparten las personas o los actores sociales. La tecnología, especialmente en la comunicación y la información, ha llegado a ser parte esencial de las condiciones, que menciona Habermas (2001, pp. 30-31). Con esto queremos subrayar que es un fenómeno reconocido y considerado por una comunidad de sujetos, y de las actuaciones comunicativas con las que se entienden; en otros términos -siguiendo el planteamiento habermasiano-, la tecnología

2 Habermas (2001, p. 30) señala: "El mundo sólo cobra objetividad por el hecho de ser reconocido y considerado como uno y el mismo mundo por una comunidad de sujetos capaces de lenguaje y acción. El concepto abstracto de mundo es condición necesaria para que los sujetos que actúan comunicativamente puedan entenderse entre sí sobre lo que sucede en el mundo o lo que hay que producir en el mundo. Con esta práctica comunicativa se aseguran a la vez del contexto común de sus vidas, del 'mundo de la vida' que intersubjetivamente comparten. Este viene delimitado por la totalidad de las interpretaciones que son presupuestas por los participantes como un saber de fondo. Para poder aclarar el concepto de racionalidad, el fenomenólogo tiene que estudiar, pues, las condiciones que han de cumplirse para que se pueda alcanzar comunicativamente un consenso". 
forma parte esencial de su práctica comunicativa con la que se asegura el contexto común de sus vidas.

Hay una consideración adicional que se debe tomar en cuenta y es que un rasgo fundamental de la experiencia de compartir intersubjetivamente el mundo de la vida hoy se extiende a la aldea global de McLuhan, con la utilización de las tecnologías de la comunicación y de la información (TIC), en especial Internet. Ciertamente, como lo expresan Fernández y Pérez (1995), los enormes progresos tecnológicos aplicados a los medios de comunicación (enlaces por satélites, los massmedia) no sólo han ampliado la cobertura inusitadamente, sino también, la instantaneidad.

Esto plantea nuevas y significativas dimensiones al ámbito de las teorías de la comunicación. Resulta impensable la infinidad de incidencias en el mundo subjetivo, el mundo objetivo de los sujetos sociales y el conjunto del sistema social resultantes de las interacciones, aplicaciones y posibilidades que se han producido y se producen en forma expansiva con las innovaciones tecnológicas.

La complejidad creciente en que se genera la dinámica personal y social de los sujetos no escapa a la realidad de las determinaciones e influencias del envolvente influjo de las innovaciones tecnológicas. "Las informaciones que provienen de las ciencias experimentales estrictas -expresa Habermas (2001, pp. 117-116) - sólo pueden entrar en el mundo social de la vida por la vía de su utilización técnica, es decir, como saber tecnológico: sirviendo entonces a la ampliación de nuestro poder de disposición técnica". La tecnología se ha constituido en un símbolo de la modernidad tardía y a la vez en un paradigma, un marco de referencia, para los sujetos sociales en las sociedades capitalistas avanzadas y subdesarrolladas.

Esto ha alcanzado tal magnitud, que las formas de control ideológico más "metafísicas" han dado paso a las formas "tecnocráticas", según la tesis de Habermas. De este modo, muchos teóricos de la ideología la demarcan epistemológicamente como sinónimo de justificación racional, técnica y "científica" para la dominación social, más que de justificaciones míticas, religiosas o metafísicas (Eagleton, 1997). Con carácter anticipatorio, Marcuse (1972) señalaba a mediados de la década de 1950, que la tecnología se había constituido en un efectivo instrumento de dominación al servicio de la legitimación de un poder político expansivo que permeaba todas las dimensiones de la cultura ${ }^{3}$. Al punto que le confirió la dimensión de un proyecto

3 La racionalidad tecnológica ha devenido en un producto superior de la sociedad racional, heredera de la Ilustración, que respalda la legalidad y legitimidad del dominio (que incluso abarca las libertades) sometidas a una concepción instrumentalista de la vida. 
histórico-social en el que se manifiestan los intereses dominantes que en la sociedad se orientan respecto de los hombres y las cosas.

La muestra más representativa del auge tecnológico y cómo ha influido en la cultura contemporánea es la creación de Internet: un sistema tecnológico basado en una conexión informática. La incidencia de esta red ha sido de tal magnitud en el mundo contemporáneo que ha creado su propia cultura: la cultura Internet. Una cultura que es el resultado de la herencia de la Ilustración y la modernidad.

En referencia a la cultura Internet, Manuel Castells (2002, pp. 57-58) observa que los sistemas tecnológicos se producen socialmente y vienen determinados por la cultura: "un conjunto de valores y creencias que conforman el pensamiento". Es la cultura de los productores/usuarios y la de los consumidores/usuarios: una construcción colectiva que trasciende las preferencias individuales que incide en las personas que participan de esa cultura (en el caso de Internet, los usuarios/productores). Castells expresa que Internet:

Muestra la capacidad de las personas para trascender las reglas institucionales, superar las barreras burocráticas y subvertir los valores establecidos en el proceso de creación de un nuevo mundo. A su vez sirve para respaldar la idea de que la cooperación y la libertad de información puede favorecer la innovación en mayor medida que la competencia y los derechos de propiedad (Castells, 2002, p. 25).

La ideología aparejada al desarrollo tecnológico ha devenido, en efecto, en un gran marco de referencia, configurando una de las más representativas tendencias en el horizonte cultural del mundo de hoy: el ethos tecnológico, que ha "modelado" una mentalidad y una actitud vital que se manifiesta de distintas maneras en las esferas interior y exterior del sujeto social y en diversos ámbitos del sistema social. Esto se manifiesta, por una parte, en la mutación de no pocas prácticas sociales (y en la aparición de nuevas) en el sistema social y, por otra, en los acentuados cambios de las relaciones sociales de producción, en la esfera del sistema económico.

Los cambios educativos, ya sea como reformas o innovaciones, se han visto afectados por esa tendencia asumiendo la racionalidad instrumentaltecnocrática que se manifiesta en las nuevas formas de tecnología educativa y en las innovaciones educativas. De acuerdo con nuestro punto de vista, el auge tecnológico, que contextualiza e incide en la irrupción y orientaciones 
de las reformas e innovaciones educativas, está marcado por cuatro grandes procesos interrelacionados.

$1^{\circ}$ La tercera revolución científico-tecnológica. El avance de los productos derivados de este proceso, que marcó el llamado largo boom de la economía, definió un proceso de avance en todas las esferas de la sociedad global, desde el ámbito micro hasta el macrosocial. Los avances científicotecnológicos que han tenido lugar, en lo que Ernest Mandel (1986) llamó la Tercera Revolución Tecnológica -ocurrida en la onda económica larga que sobrevino después de la Segunda Guerra Mundial-, han colocado el concepto de innovación en un lugar privilegiado en el lenguaje contemporáneo. Conceptos tales como: informatización de la sociedad, sociedad de la información, sociedad red, sociedad empresa, son claras expresiones de la nueva racionalidad instrumental del mundo capitalista avanzado, y conspicuas manifestaciones del progreso técnico y su aplicación al mundo social de la vida en que se despliegan las innovaciones tecnológicas. Ellas inciden en las transformaciones estructurales de las sociedades capitalistas industriales avanzadas de las que, al mismo tiempo, son producto. El uso de este término está delimitado a aplicaciones del conocimiento derivado de la ciencia normal ${ }^{4}$ y su aplicación científico-tecnológica en diferentes campos: astronomía, informática, robótica, física atómica, mecánica cuántica, biotecnología, tecnología de la información y la comunicación (sin duda la creación y desarrollo de Internet es una muestra ostensible del enorme avance que ha experimentado la cultura humana resultante de la revolución científico-tecnológica), y en otros más, por ejemplo, en investigación y desarrollo (I+D).

En el ámbito educativo, las aplicaciones científicas al desarrollo tecnológico influyeron en la aplicación de la tecnología a la educación, como es el caso de la pedagogía tecnológica o tecnología de la educación. Esta influencia se puso de manifiesto en la aplicación de los resultados de las investigaciones psicológicas a los procesos de aprendizaje: conductismo, psicología cognitiva, Gestalt... Esta pedagogía parte de la premisa de "que si es posible tener una tecnología de la producción, también es posible tener una tecnología de la instrucción" (...) "poniéndole más atención a los medios que a los contenidos" (Ordóñez, 2004, pp. 165-166). La tecnología educativa ha mostrado también un notable avance con la introducción de la tecnología aplicada al campo de la organización escolar, introduciendo nuevos modelos tecnológicos

4 Concepto expuesto por Kuhn (1986) en su obra la Estructura de las revoluciones cientificas. Chalmers (2000) señala en esta perspectiva que la actividad de la ciencia normal se desarrolla a partir de un paradigma científico como guia de la investigación y la interpretación de los fenómenos observables. 
de funcionamiento de los procesos de enseñanza y aprendizaje (p. ej. en el desarrollo curricular) y modelos organizacionales. Asimismo, existe un inusitado avance de las TIC aprovechando el enorme potencial de Intemet.

$2^{\circ}$ La emergencia de la ideología tecnocrática. La controversia mundial entre la razón política y la razón técnica efectuada a finales de la década de los cincuenta e inicios de los sesenta se generó alrededor del concepto de tecnocracia: un fenómeno ligado al interés por la eficiencia, la competencia profesional en la esfera de la toma de decisiones gerenciales y al progreso técnico. La visión tecnocrática se consolida en el mundo capitalista moderno, a raíz de las condiciones estructurales originadas en la creciente especialización de los avances científico-tecnológicos en los procesos de producción industrial y el desarrollo de las industrias, como unidades empresariales que conforman el sistema industrial. En esta tesitura, las tendencias a la concentración de las empresas (en la lógica de consolidación del capital monopolista) y las crecientes y complejas acciones empresariales desencadenan la proliferación de planes generales y programas empresariales, constituyendo un sistema de provisiones, cuya finalidad es conferirle racionalidad al desarrollo económico. El desarrollo de las innovaciones tecnológicas en el campo de la gestión empresarial, desde las expresiones del fordismo y taylorismo, abarcó el ámbito de la gestión educativa en la visión organizacional y de la gestión escolar creando el técnico de la enseñanza. Estos modelos han incidido en los procesos de administración escolar configurando una ideología de la administración escolar, que responde a una lógica empresarial en la organización y el control de los centros educativos, que adoptan sus normas y prácticas laborales (Apple, 1997).

La instauración de la tecnocracia trajo aparejada la ideología tecnocrática, que asume la racionalidad técnica e instrumental basada en la preeminencia de la eficiencia y la competencia, y la sobrevaloración de la profesionalización de las decisiones gerenciales al más alto nivel, es decir, en el “ápice” de la estructura organizacional de las grandes empresas. El fenómeno tecnocrático trajo consigo, en las sociedades industriales, la figura del tecnócrata, el generalista, el manager, que ocupa ese lugar en el nivel más alto del proceso de producción industrial; como director supremo de ese proceso, que utiliza los técnicos o especialistas (los expertos de lo particular), pero que coordina los resultados de la colaboración de los demás y los integra en

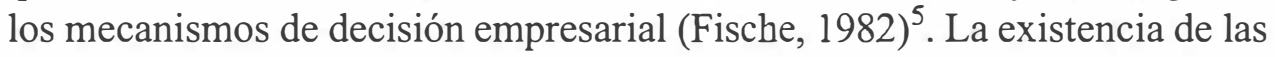

5 Como grupo social los tecnócratas desempeñan un papel estratégico en los procesos de transición o de cambio estructural (como las crisis de acumulación) preconizando la racionalización de la concepción técnicoinstrumental de la vida social, como ideología, especialmente en las crisis de legitimación. Su accionar se 
tecnoélites, como resultado de la cultura meritocrática asociada a la apertura tecnológica que tuvo lugar con la emergencia de Internet (Castells, 2002), es una muestra de la evolución que ha experimentado el fenómeno tecnocrático estrechamente vinculado con el desarrollo de la estructura tecnoproductiva, como expresión del desarrollo vertiginoso de las fuerzas productivas en los países de capitalismo tardío.

La cultura Internet ha creado una cultura meritocrática, en la cual el mérito se mide por la contribución del desarrollo de un sistema tecnológico fundado en la idea modernista de que el desarrollo científico y tecnológico es un componente clave de la humanidad. El influjo de las innovaciones tecnológicas no sólo ha incidido profundamente en el campo educacional, sino que ha promovido la constitución de un enorme contingente de tecnócratas educativos, que están al servicio de los grandes organismos internacionales y think tanks al servicio del capitalismo global y su ideología neoliberal (Banco Mundial, Fondo Monetario Internacional, Organización Mundial del Comercio, Organización para la Cooperación y el Desarrollo Económico (OCDE), Council of Foreings Relations de los Estados Unidos, etc.).

$3^{\circ}$ Los cambios en la estructura tecnoproductiva. La crisis de acumulación de capital de la década de los setenta puso de relieve en los años siguientes, que la generalización de la aplicación de las innovaciones tecnológicas a la esfera de la producción ha sido fundamental en el crecimiento espiral de la producción y los beneficios de capital, como lo fue en el periodo de posguerra. La innovación tecnológica demostró ser un factor básico para el aumento de las composiciones orgánica y técnica del capital, como de los métodos y técnicas organizacionales de producción. Desde el punto de vista macroeconómico y social, los cambios tecnológicos en el proceso de producción que acompañan la acumulación de capital (resultantes de la introducción de la nueva base tecnológica) modifican las formas de organización y el trabajo. En consecuencia, las innovaciones tecnológicas aplicadas a la economía informacional se han constituido en un factor esencial en el vertiginoso avance que han experimentado la productividad y la competitividad económicas y el crecimiento de la producción industrial masiva. Con lo cual se inicia en el sis-

centra en promover y ejecutar las grandes formulaciones estratégicas y los grandes objetivos de las políticas estatales y sus formulaciones programáticas, en el marco de esa visión. Una clara manifestación de la ideología tecnocrática fue, por ejemplo, la irrupción de la visión modernista y los programas desarrollistas que sirvieron de marco de referencia a los planes estatales, en las décadas de los cincuenta y los sesenta (en los cuales educación ocupó un lugar primordial). Los tecnócratas han desempeñado un papel fundamental en la divulgación y transferencia de la visión tecnocrática empresarial a otras esferas de la gestión pública, entre las cuales están las reformas e innovaciones educativas. 
tema social, un proceso de ajuste funcional y operativo en el funcionamiento del sistema económico, del sistema institucional y del sistema cultural (especialmente de sus pautas). Esto provocó un proceso de acomodación estructural del sistema en su conjunto, frente a la crisis de acumulación para adaptarse a las nuevas necesidades reproductivas que exige el cambio tecnológico en el conjunto del proceso productivo. Un notable ejemplo de esto es el desarrollo de la economía informacional, en la era del globalismo neoliberal, que ha devenido en un factor esencial en el vertiginoso avance experimentado por la productividad y la competitividad económicas ligadas a la expansión del capital transnacional.

Los cambios educativos propuestos, como parte de la política económica neoliberal, se han originado en buena medida como reacción a las demandas reproductivas de la fuerza de trabajo ligadas a la ampliación de las necesidades tradicionales de reproducción, y a las nuevas necesidades surgidas por las transformaciones tecnológicas y las modificaciones sistémicas en los métodos de producción. Las demandas de cualificación de las fuerzas de trabajo emergentes, acompañadas de las modificaciones en la cultura del consumo (cambio de los patrones cualitativos y cuantitativos de consumo de los trabajadores), conjuntan un cuadro estructuralmente articulado que incide en los cambios en la estructura tecnoproductiva.

$4^{\circ}$ EI tecnoglobalismo. La internacionalización de la transmisión y difusión de las tecnologías ha sido una condición de la unificación económica en ámbitos como el comercio, los procesos de integración y la constitución de las redes del mercado de capital. El tecnoglobalismo es un rasgo distintivo de la globalización económica que posee tres grandes significados. $1^{\circ} \mathrm{La}$ creciente explotación de innovaciones tecnológicas en los mercados internacionales, que se concreta a través de diversas vías como el comercio de productos de alta tecnología o mediante la explotación de patentes. $2^{\circ} \mathrm{La}$ tendencia al incremento de la cooperación internacional en materia tecnológica, que se manifiesta en el aumento de países que comparten los nuevos conocimientos técnicos. $3^{\circ} \mathrm{La}$ generación internacional de tecnologías vinculada a las empresas multinacionales y transnacionales. Las redes de transferencia tecnológica, la divulgación del conocimiento tecnológico (Requeijo, 2000).

\section{Reforma e innovación educativas}

Tanto la reforma como la innovación educativa son subcategorías generales de una categoría más amplia: el cambio educativo. La visión dialéctica 
de la hermenéutica crítica que hemos venido desarrollando presupone, epistemológica y metodológicamente, de un análisis ideológico de la intertextualidad entre las formulaciones discursivas de la política educativa y curricular y los cambios estructurales del sistema social, como referentes concretos, en cuya interacción dialéctica surge el cambio educativo. A este respecto, hemos introducido los conceptos de teleología explícita y teleología concreta ${ }^{6}$ para designar los dos momentos de formulación de los objetivos educacionales (como estrategia de legitimación de determinados intereses políticos), en las diversas formulaciones discursivas de la política educativa y las prácticas socioeducativas, que se realizan en el contexto que delimitan estructuralmente. Todo proceso de cambio educativo es parte integrante de un proceso de cambio estructural y, por lo tanto, representa un proceso de estructuración ideológica, que se expresa en una formulación discursiva de política de educación (teleología explícita), en la cual se oculta un conjunto de intenciones políticas no explícitas, pero que representa el contenido real del cambio en su orientación política (teleología concreta). Veamos algunas de las principales características de ambas expresiones del cambio político educacional.

\section{Reforma educativa}

Según la visión hermenéutica crítica que hemos asumido (Miranda, 2006), las reformas obedecen a una estrategia de legitimación de un determinado sistema hegemónico político y cultural en la sociedad global. De partida, hay que considerar que la reforma como cambio a gran escala afecta la política educativa de la que emana, y los fines, objetivos, estrategias y prioridades del sistema educativo, en su conjunto. Asimismo, las reformas se desarrollan en el contexto sociopolítico, cultural e institucional, del cual es parte integrante el sistema educativo, en consecuencia, la reforma educativa posee una dimensión general que se realiza en un nivel macro, es decir, abarca la estructura del sistema educativo o revisión o modificación del currículo (González y Escudero, 1987, p. 13). Si existe un rasgo distintivo en el cambio educativo, es su multidimensionalidad. Toda reforma educativa implica cambios o innovaciones concretas, que pueden ser aceptadas sin discusión cuando en su proceso de aprobación legislativa ha prevalecido el consenso o

6 Hemos escrito dos ensayos sobre este tema: Hacia una visión hermenéutico critica de la politica educativa, cfr. Miranda (2006), y "Política curricular, crisis de legitimación y hegemonía neoliberal. Una visión desde la sociología de la educación crítica” (en el cual introducimos los conceptos de teleología explícita y teleología concreta) próximo a publicarse en la Revista de Ciencias Sociales de la Universidad de Costa Rica. 
una negociación o concertación que comprende los intereses corporativos y la cultura profesional de los docentes, o cuando los criterios de los "expertos" no encuentran resistencias en los diversos actores de la sociedad civil (Viñao, 2002, p. 109).

Por su carácter de cambio educativo a gran escala, la reforma educativa implica una estrategia planificada para la modificación de aspectos concretos del sistema educativo de un país, definidos con arreglo a un conjunto de intereses sociopolíticos y/o económicos, de necesidades, que persigue resultados específicos a través de medios y métodos adecuados y eficaces. Por lo tanto, las reformas educativas corresponden a una política de educación e implica un conjunto de objetivos, de directrices y un modelo de estructuración de un país determinado. La reforma está concebida para provocar variaciones a gran escala en el marco de la enseñanza, metas, estructuras u organización (García, 1998). Al igual que la innovación educativa, abarca al menos las siguientes dimensiones de cambio educativo, como formulación política al más alto nivel de decisiones estatales (ámbito de las políticas públicas): intencionalidad simbólica, contenido axiológico, nivel de formulación y/o diseño, ámbito de cambio educativo, concepción sobre el cambio educativo, dimensión práctica en el sistema educativo (González y Escudero, 1987).

\section{- Intencionalidad simbólica}

Desde el punto de vista de la intencionalidad simbólica, toda reforma educativa tiene una naturaleza ideológico-política, es la expresión de los intereses de clase, grupos sociales; es la expresión de un proyecto políticoeconómico formulado, por lo general, en un programa de gobierno de un determinado partido político, que en ejercicio del gobierno (clases dirigentes), promueve un modelo de sociedad, de desarrollo social, de sujetos sociales, etc. En síntesis, la reforma educativa tiene un contenido político-ideológico, representa y es parte integrante de un programa político y económico de las clases dirigentes y otros grupos sociales, sus partidos políticos en un contexto nacional.

La reforma es una cuestión de ideales nacionales (estén o no estén sus consecuencias conscientemente compartidas por todos los ciudadanos), un acto de afirmación ideológica, que se suele mover a través de y con la poderosa retórica de la modernidad, del bien común o del Estado de bienestar. El concepto de reforma denota solamente la estructura de dicha retórica, pero no tiene que suponer el cambio en los patrones de conducta social (Angulo, 1994, p. 365). 


\section{- Contenido axiológico}

Como propuesta de cambio educativo, la reforma comprende en su configuración teórica y doctrinal un conjunto de valores políticos, sociales, culturales e ideológicos de las doctrinas políticas y los programas políticos en que se inscriben. Representa un proyecto educativo de cambio expresado en fines, principios y orientaciones. Tanto la intencionalidad simbólica como el contenido axiológico son partes integrantes de la teleología explícita.

\section{- Nivel de formulación y/o diseño}

Como resultado y producto de la política educativa estatal, la reforma educativa obedece a un proceso de planificación como parte de una estrategia de legitimación de los contenidos de la teleología explícita. En consecuencia, como expresión de la política educativa requiere de medios e instrumentos que posibiliten su implantación en el medio social. En este proceso intervienen el gobierno central, los subsistemas del sistema educativo y administraciones educativas. Cuando la reforma es de "abajo hacia arriba", supone la participación, en algún grado, de los integrantes de la comunidad educativa y de los actores sociales o grupos de interés de la sociedad civil, vinculados directa o indirectamente con el sistema educativo. Por el contrario, si es de "arriba hacia abajo", obedece a una estrategia política de las clases dirigentes por establecer una hegemonía política y cultural, utilizando medios legales formales, pero no de consulta democrática de consenso amplio.

\section{- Ámbito del cambio educativo}

Por su naturaleza de incidir a gran escala, la reforma está estrechamente ligada a los cambios estructurales en los que pretende incidir, con una clara intencionalidad (teleología concreta), el Estado (y, por supuesto, las clases dirigentes, a través de él) por medio de la política educativa. Por lo tanto, la incidencia en el sistema social, particularmente la sociedad civil (sujetos sociales, actores sociales, organizaciones privadas, grupos y movimientos sociales, de distinta naturaleza, etc.), es la razón fundamental que delimita el ámbito de la reforma de acuerdo con su intencionalidad simbólica.

\section{- $\quad$ Concepción sobre el cambio educativo}

Se enmarca en un determinado enfoque acerca del cambio educativo: qué son y cómo funcionan los procesos de cambio educativo, qué variables inciden estos y cuáles procesos se desarrollan para producir cambios en situaciones educativas de distinta proporción. La categoría de cambio 
educativo, como lo hemos apuntado, tiene un amplio significado que no sólo denota, por su naturaleza genético-estructural (es decir de su vinculación estructural concreta con la realidad sociohistórica), su magnitud en el sentido de su alcance social, sino también otras dimensiones como su orientación ideológico-política, los fundamentos epistemológicos educativos, las teorías pedagógicas, abarcando todas las formulaciones curriculares y las prácticas educativas concretas, que se manifiestan en lo que Postic (1982) ha conceptualizado como relación educativa?

\section{- Dimensión práctica en el sistema educativo}

Se desarrolla a partir de un determinado tipo de práctica en el interior del sistema educativo, que se manifiesta en procesos y metodologías de aplicación socioculturales, tecnológicas, institucionales, métodos pedagógicos, enfoques curriculares, construidos a partir de marcos de referencia sociopolíticos e ideológicos, teorías educativas y mediante una determinada mediación pedagógica. Es el ámbito de lo que Pérez Gómez (1999) ha llamado cultura institucional: el espacio donde se realizan las interacciones que se producen en el contexto escolar, contexto además donde las prácticas escolares son acomodadas por las diversas instancias administrativas de acuerdo con las exigencias del escenario político y económico de cada época y lugar.

\section{La innovación educativa}

La innovación educativa tiene un alcance menor que las reformas y se realiza en culturas escolares determinadas. Examinaremos, en primer lugar, algunas dimensiones básicas del concepto de innovación para comprender su significado e iniciar un análisis crítico de su aplicación en el ámbito educativo. Innovación puede significar creación de algo nuevo, invención en la cual dos elementos o entidades se combinan de forma novedosa produciendo una nueva configuración, o puede ser una modificación de la preexistente. Esto se debe complementar con dos aspectos: $1^{\circ}$ Debe existir la percepción y corroboración de que eso nuevo se haga parte del acervo cognoscitivo de los usuarios y de su aplicación concreta; y, $2^{\circ}$ el carácter de una innovación está

7 Acerca del concepto de relación pedagógica Marcel Postic (1982, pág. 10) observa: "Ningún pedagogo puede, de ahora en adelante, abordar el análisis del acto pedagógico solamente en términos de contenidos y de métodos. Se han diseñado orientaciones de estudio; en lugar de concebir la relación educativa bajo la forma de una comunicación entre el alumno y el enseñantc en medio de la masa de los alumnos de la clase, la escuela y la sociedad, las relaciones de los sujetos con el saber y la cultura". 
definido como una idea, una práctica o un objeto material que es concebido como una "novedad", independientemente que se acepte o no, que implica la "asimilación" de lo nuevo (Zaltman, Duncan y Holbeck, citados en Angulo, 1994).

La innovación es el resultado de un proceso creativo para perfeccionar o renovar algo, en consecuencia, la introducción y puesta en práctica de la innovación supone mejorar, en un aspecto específico o en su conjunto, aquello que se persigue modificar. La innovación se asocia a fines o intenciones que le confieren sentido, generalmente ligados a la idea de un avance hacia el progreso por la vía del desarrollo técnico que se expresa en el concepto de progreso técnico. Los procesos que se conceptualizan como innovaciones en su sentido genérico responden en la práctica a una necesidad de modificación o alteración de un estado de cosas; por lo tanto, se efectúan en la lógica de un proceso de creación, de transformación o de invención de uno o de varios aspectos del objeto o la realidad que se pretende modificar, reformar o transformar. Si bien la innovación responde a un proceso intencionado, puede ser también el resultado de una transformación accidental o inintencionada. En todo caso, hay que agregar a lo anterior que la innovación tiene como precondición un "estado de avance" del conocimiento y de sus aplicaciones tecnológicas que son parte esencial del contexto en que se construyen las innovaciones (Angulo, 1994).

Con base en las consideraciones precedentes, queremos destacar el hecho singular de que la política educativa adquiere, por lo general, una expresión de ejecución y aplicación concreta, por medio de la implementación de innovaciones educativas. Estas se constituyen en elementos mediáticos entre las reformas educativas, las políticas educativas, la normatividad educativa (dimensión macro) y las diversas prácticas educativas concretas en los centros escolares (dimensión micro). Las innovaciones educativas poseen un sentido transformador de la realidad educativa, por su carácter más específico, orientado a promover una mayor eficacia en el cumplimiento de los objetivos del sistema educativo.

Estos aspectos mencionados se presentan en su formulación discursivo-técnica como aparentemente neutrales. Esta apariencia de neutralidad respaldada por lo técnico hace que el medio educativo sea valorado como aséptico, esto es, despojado de toda intencionalidad ideológico-política. En consecuencia, se despoja la innovación de su vinculación con otros factores fundamentales sociopolíticos. Es común ver cómo este error se ha generalizado en la cultura académica, y hoy es una creencia firme de la que participa 
una gran cantidad de educadores, e incluso docentes universitarios del campo educativo. Como lo puntualizan González y Escudero (1987, p. 21), en virtud de que las innovaciones se construyen e implementan al amparo de contextos sociopolíticos e ideológicos que las legitiman poseen un carecer político; el cambio curricular es, en efecto, parte de un espacio más amplio como lo es el sistema social; por consiguiente, no es neutral en un sentido político e ideológico. Sin embargo, según nuestra visión, hay que considerar que si bien las reformas e innovaciones son legitimadas, al mismo tiempo, se constituyen en instrumentos legitimadores fundamentales, como partes integrantes de las estrategias de legitimación, que se llevan a cabo en contextos de crisis (crisis de acumulación de capital, de legitimación, crisis sistémicas transferidas, etc.).

Por el ámbito del cambio educativo la diferencia entre reforma e innovación educativas radica en que mientras la reforma, por su dimensión global, se orienta a realizar cambios fundamentalmente estructurales, las innovaciones educativas se relacionan con los procesos educativos y los contextos en los que funcionan y operan las transformaciones concretas (González y Escudero, 1987). Así pues, la esfera de aplicación y concreción de las innovaciones educativas es en el ámbito microeducativo, en los contextos inmediatos de funcionamiento de los procesos pedagógicos. Por ello, supone un cambio en las prácticas educativas. En esto radica su importancia, pues incide de manera directa en los cambios educativos que se persiguen.

González y Escudero (1987, p. 16) definen la innovación como "la serie de mecanismos y procesos más o menos deliberados y sistemáticos por medio de los cuales se intenta introducir y promocionar ciertos cambios en las prácticas educativas vigentes". A su vez, destacan el carácter novedosọ de las innovaciones para referirse " a dinámicas explícitas que pretenden altérar las ideas, concepciones, metas, contenidos y prácticas escolares, en alguna dirección renovadora de la existente". Por su dimensión específica en el proceso de cambio educativo, la innovación persigue incidir en aspectos puntuales de la práctica educativa. Aunque es preciso reconocer que esas renovaciones o mejoras educativas puntuales son manifestaciones de un proceso de cambio, por lo general, suficientemente encubierto de otras intenciones no explícitas ni manifiestas, y que trascienden la dimensión exclusiva de lo educacional; esto corresponde al ámbito de la teleología concreta. Coincidimos con González y Escudero cuando afirman que: "El sentido del cambio, el quien lo promueve el cambio y el para qué del mismo son aspectos sólo comprensibles desde una lectura sociopolitica de la innovación" (1987, p. 21). 
Motivada por diversos factores de índole tecnológica, política, personal, institucional, etc., la innovación se orienta a provocar cambios en la práctica educativa: metas y estrategias de enseñanza, patrones organizativos, etc. Existen diversas clasificaciones de la innovación, de acuerdo con los objetivos de cambio educativo que se planteen modificar.

Veamos dos clasificaciones (referidas por González y Escudero, 1987, p. 18) que reconocen nueve dimensiones de la innovación curricular: 1) presupuestos de base y aspiraciones; 2) objetivos; 3) conductas de entrada de los alumnos; 4) contenidos; 5) materiales instructivos; 6) estrategias de enseñanza; 7) experiencias de aprendizaje; 8) tiempo; y 9) instrumentos y procedimientos de evaluación. Fullan y Pomfret (ídem.), por su parte, destacan cinco aspectos básicos de la innovación: 1) cambios en asignaturas y materiales, referidos al currículo; 2) alteraciones estructurales en la organización formal y el espacio físico en el que se realiza la enseñanza, provocando cambios en las condiciones en que se establecen las interacciones de los sujetos receptores de la innovación; 3) cambios conductuales o manifestaciones conductuales del cambio en las funciones o relaciones de los sujetos (estilos de enseñanza, nuevas tareas y funciones para apoyar estilos: desarrollo curricular, planeamiento de los educadores...); 4) conocimiento y comprensión de la innovación, en aspectos tales como: filosofia, valores, presupuestos, objetivos, estrategias de puesta en práctica, etc.; 5) internalización del cambio, relativa a la necesidad de una conciencia del cambio y una valoración positiva y compromiso para llevarlo adelante.

Estos ejemplos son sólo representativos de una abundante existencia de tipologías de innovaciones. Nos parece de cardinal importancia resaltar que el análisis de las innovaciones no se agota en estos aspectos explícitos; por el contrario, deben ser sometidos al análisis de fondo de la hermenéutica crítica para trascender la comprensión como manifestaciones fenomenológicas de la esencia que se oculta en esas dimensiones; este es el salto dialéctico cualitativo de la teleología explícita a la teleología concreta de las innovaciones educativa (Miranda, 2006). Debe señalarse que tanto las reformas como las innovaciones educativas producen -como lo indica Viñao (2002, pp. 110-113)- efectos no previstos, queridos o buscados. Puede suceder que en algunos casos tengan lugar comportamientos o actitudes totalmente opuestas a las que se pretendían en principio. A este respecto, Rudduck manifiesta que "los esfuerzos para introducir cambios han tendido a subestimar el poder de la cultura y del aula para adaptar, aceptar y rechazar innovaciones que entran en conflicto con las estructuras y valores dominantes en la cultura escolar"8 (1994, p. 387).

$8 \quad$ El destacado aparece en el texto original. 
Suele suceder, asimismo, siguiendo a Viñao (2002), que los tres ámbitos en que se manifiestan las innovaciones: la teoría (las propuestas), la legalidad (prescripciones) y las prácticas (las relaciones educativas en el aula y los centros educativos), podrían tener contrastes que impidan u obstaculicen la innovación, entre los cuales cabe mencionar:

- Lenta difusión o no aceptación de las innovaciones que encuentran resistencias u obstáculos por los agentes o actores que las deberían aplicar.

- Permanencia de continuidades, tradiciones más allá del reconocimiento legal; la necesidad de conocer y analizar los procesos de construcción, consolidación y cambio de las prácticas cotidianas en el aula y fuera de ella.

- Oposición en tres culturas escolares con puntos de vista y preocupaciones distintas: la cultura de los reformadores y gestores de la educación (políticos, administradores, supervisores), la cultura de los especialistas en el campo educativo (profesionales universitarios, centros de investigación educativa) y la de los profesores y maestros.

- Resistencia a la introducción de innovaciones tecnológicas, metodológicas, modelos de organización escolar y estrategias de enseñanza y aprendizaje.

- La reacción a la incidencia de las innovaciones educativas, principalmente de naturaleza curricular, en los procesos de cambio estructural en el sistema social.

No obstante, nos parece, que el análisis de Viñao (2002), debería complementarse con otro tipo de efecto no previsto, pero que se manifestaría en un plano más general y que situamos en el plano de la teleología concreta: el de la ineficacia de la innovación en el marco de su papel en una estrategia de legitimación (como parte integrante de un proceso más amplio de reforma educativa, o considerando un interés estratégico de alto nivel que podría tener): Nos referimos al hecho de que por diversas razones, la innovación, en cuenta las mencionadas, no logre incidir eficazmente en las reales y ocultas intenciones de cambio en los procesos estructurales que tengan lugar en el sistema social: las de naturaleza sociopolítica e ideológica.

Sucede en el campo de aplicación de las innovaciones pedagógicas que en el aula, dimensión nuclear, es donde tiene lugar la relación pedagógica (entre el o la docente y los y las estudiantes), y, en paralelo, se ponen de manifiesto la relación entre la teoría y la práctica, las diferencias entre 
los objetivos generales de la reforma y su concreción por medio de las innovaciones educativas. Hay reformas que, en efecto, favorecen la innovación y otras que la entorpecen e incluso la obstaculizan. Un ejemplo representativo de esta cuestión podría ser el que refiere Laval (2004, pp. 260-261), que se efectuó en los Estados Unidos en la década de 1920, con la introducción de principios de gestión empresarial científica, aplicados al ámbito de la administración, la organización y la cultura organizacional educativa: la de los "administradores escolares" formados en el espíritu empresarial e industrial norteamericano de la eficiency.

La gestión de la enseñanza que se gestó en esa década y la formación especial en institutos especializados colocaron a la función administrativa de la cultura y los valores de los educadores en un lugar central. El papel de este tipo de formación sirvió para forjar una generación de líderes empresariales mediante la gestión de esos administradores, que se impusieron en las instituciones educativas casi como "verdaderos patronos". Esos expertos desempeñaron una función social y cultural de primer orden como intelectuales orgánicos en la configuración y consolidación de la cultura hegemónica de los grandes capitalistas industriales norteamericanos.

Según Laval (2004, pp. 260-261), el distanciamiento del contenido cultural (dimensiones éticas, morales o científicas de la formación) de los estudios obedeció, en ese periodo, a los cánones de la lógica empresarial y de sus valores de los business condicionados por la racionalización taylorista y por las presiones económicas. Aunque la depresión económica de 1929 (surgida a raíz de la crisis de acumulación de capital), socavó las bases de esa tendencia, mostró que ya no se podía exaltar la eficacia social; las posiciones de poder se habían consolidado en las escuelas y particularmente en los institutos de formación del profesorado, por los expertos que habían adoptado el ethos de la gestión empresarial.

Es importante destacar que la introducción de ese tipo de innovación taylorista incidió y predeterminó la "profesionalización de la enseñanza", dando paso al surgimiento de un nuevo tipo de educador: el "técnico de la enseñanza", "los simples ejecutantes encargados de aplicar los innovaciones $y$ de poner en funcionamiento los procedimientos normalizados de la enseñanza". Este representó la contraparte desde la óptica taylorista de los expertos en ciencias de la educación. Los cambios surgidos por la búsqueda de las economías de producción a gran escala y la masificación del consumo indujeron a cambios educativos que hicieron aumentar el tamaño de las aulas y a eliminar, por un criterio de rentabilidad, los pequeños grupos y 
las materias que se consideraban "inútiles". La racionalización por bajar los costos educativos significó la reducción de los contenidos y las exigencias culturales. El ejercicio docente, por su parte, se burocratizó al adecuarse a esa nueva concepción educativa: el nuevo "técnico de la enseñanza" subordinó su práctica docente de transmisión de conocimientos por el sistemático examen de los tests, por los cuademos de seguimiento y por la actualización de las estadísticas.

Este ejemplo es ilustrativo de cómo la reforma educativa referida, acompañada de sus correspondientes innovaciones, afectó la cultura organizacional-educativa, el currículo y, en general, las prácticas educativas obedeciendo a una estrategia más amplia: un proyecto de acción sociopolítico y económico que expresaba los intereses de ciertos grupos, a un proyecto global de transformación que se promovía para implantar una racionalidad técnico-instrumental acorde con la imperiosa necesidad de lograr un relanzamiento de la acumulación de capital ante la crisis de la década de los treinta.

La formación de un contingente de fuerza laboral altamente capacitado para atender las demandas de la nueva estructura ocupacional y la nueva estructura social de acumulación que requería el sistema capitalista en los Estados Unidos, en aras de una modemización de su estructura en el contexto de la crisis. La configuración de un tipo ideal de fuerza de trabajo para atender la crisis y las nuevas demandas del sistema económico se expresaban en el nuevos ethos empresarial-industrial, que requería la cultura hegemónica capitalista en crisis. El efecto producido por la aplicación de las innovaciones, en el caso de referencia, fue la formación de tecnócratas en el campo de la educación y, paralelamente, la entronización de una visión cultural tecnocrática de la educación que desplazó en cierta medida los postulados humanistas y democráticos de algunas tradiciones pedagógicas en Estados Unidos.

La respuesta de cambio educativo (de sus fines y funciones), ante la crisis de acumulación de capital de la década de 1930, implantó las bases epistemológicas, pedagógicas y socioeducativas de la corriente pedagógica de la educación tecnológica o la tecnología educativa a la que posteriormente se le integró la racionalidad psicologista, la visión de la administración educativa desde la racionalidad propia de la cultura empresarial y, consecuentemente, la racionalidad economicista-empresarial de los distintos enfoques y modelos de evaluación de la educación y de los centros educativos: assessment, accountability, appraisal...

Retomando el análisis de la relación entre la reforma y la innovación, es oportuno subrayar que ambas son complementarias. La posibilidad de los 
cambios que preconizan las reformas está mediatizada por las innovaciones, sin las cuales no sería posible llevarlas a la práctica. Los aspectos estructurales del cambio de aquellos sólo adquieren sentido cuando estos provocan en las situaciones educativas a que se destinan, cambios en la cultura escolar, en su dinámica interna, en los valores, actitudes, prácticas y marcos referenciales de todos los actores que intervienen en la relación pedagógica; y en última instancia, en los contextos socioculturales inmediatos de los centros educativos (Pérez Gómez, 1999). Al mismo tiempo, la implementación de las innovaciones influye, por medio de los resultados de su'proceso de evaluación, en la reforma, modificando o ampliando algún aspecto de su orientación, direccionalidad, prioridades y otras dimensiones de su formulación en políticas educativas. Como procesos de cambio educativo, la reforma y la innovación requieren, para hacer factible su establecimiento de un acuerdo, de una concertación, en razón de su naturaleza conflictiva y polémica y los diversos intereses de los grupos de interés que convocan (clase dirigente, partidos políticos, movimientos sociales, actores de la sociedad civil, educadores, padres, alumnos, etc.). Esto sucede con todo fenómeno innovador que inherentemente contenga hondas repercusiones sociales. Presentamos, como síntesis explicativa, en el siguiente cuadro, una perspectiva analíticocomparativa entre la reforma educativa y las innovaciones educativas.

\section{ANÁLISIS COMPARATIVO ENTRE REFORMAS E INNOVACIONES EDUCATIVAS SEGÚN DIMENSIONES DEL CAMBIO EDUCATIVO}

DIMENSIONES DEL CAMBIO EDUCATIVO

REFORMA EDUCATIVA

Intencionalidad simbólica
- Representa los ideales políticos, sociales, culturales y económicos de clases sociales, partidos políticos y del Estado. Explícita o implícitamente propone un modelo de sociedad y de desarrollo social.
INNOVACIONES

EDUCATIVAS
- Representa las dinámicas explícitas que pretenden modificar las ideas, concepciones, metas, contenidos, prácticas educativas en alguna dirección renovadora. Abarca las dimensiones intencional, estructural, pedagógica, curricular, evaluadora y otras, de la relación educativa. 
DIMENSIONES DEL CAMBIO EDUCATIVO

REFORMA EDUCATIVA

Contenido axiológico
- Valores políticos, sociales, culturales e ideológicos de las doctrinas políticas y los programas políticos en que se inscriben. Representa un proyecto educativo de cambio expresado en fines, principios y orientaciones (teleología explícita).

\section{INNOVACIONES} EDUCATIVAS

Políticas educativas estatales (medios e instrumentos) intervienen el gobierno central, los subsistemas del sistema educativo de las administraciones educativas. Cuando la

Nivel de formulación y/o diseño reforma es de "abajo hacia arriba", supone la participación en algún grado de los integrantes de la comunidad educativa y de los actores sociales o grupos de interés de la sociedad civil, vinculados directa o indirectamente con el sistema educativo.

- Cambios (estructurales) a gran escala que inciden en la

Ámbitos del cambio educativo política educativa, sus orientaciones, objetivos, estrategia y prioridades de todo el sistema educativo. Incide en el sistema social.
- Valores socioeducativos, culturales y pedagógicos del modelo sociopolítico de la educación, de la concepción sobre el cambio educativo y del enfoque teórico (pedagógico, curricular, didáctico $\mathrm{y} / \mathrm{o}$ evaluativo) sobre la innovación en que se enmarquen.
- Política curricular, instancias de decisión política (marco configurador del diseño). Planificación y diseño de programas y proyectos de innovación. Intervienen las administraciones educativas, equipo docente, etc., dependiendo del nivel de concreción.

- Cambios novedosos en la cultura escolar destinados a mejorar o modificar determinados aspectos de los procesos educativos. Transforma un ambiente, comunidad, grupo, institución, agentes y actores educativos, relación educativa, aula, etc.

- Se enmarca en un determinado enfoque acerca del cambio educativo: qué son y cómo

\section{Concepción} sobre el cambio educativo funcionan los procesos de cambio educativo, qué variables inciden en estos y cuáles procesos se desarrollan para producir cambios en situaciones educativas de distinta proporción.
- Presupone las mismas premisas, con la diferencia de que por su naturaleza más reducida, tiende a encubrir la teleología concreta, esto es, la intencionalidad real de la propuesta de cambio educativo que asume. 
DIMENSIONES DEL CAMBIO EDUCATIVO

REFORMA EDUCATIVA

- Se desarrolla a partir de un determinado tipo de práctica en el interior del sistema

Dimensión práctica en el sistema educativo educativo, que se manifiesta en procesos y metodologías de aplicación socioculturales, tecnológicas, institucionales, pedagógicas, construidos a partir de marcos de referencia sociopolíticos e ideológicos.
INNOVACIONES EDUCATIVAS
- Presupone el mismo proceso, utiliza los mismos medios y parte de los mismos fundamentos ideológicos, aunque posee un carácter más específico y una incidencia mayor en las prácticas educativas.

Fuente: Elaborado por el autor, basado en González y Escudero (1987), Angulo (1994) y aportes personales.

\section{El análisis hermenéutico crítico de modelos teóricos de las refor- mas educativas}

Toda política educativa, en su concreción de reforma o innovaciones educativas, se enmarca en uno Q varios modelos teóricos; su análisis sistemático y hermenéutico posibilita comprender las contribuciones de la educación y sus interrelaciones con los demás procesos sociales. Estos modelos tienen como función ideológica básica orientar y establecer las bases de los cambios del sistema educativo; por ello, desempeñan una función de mediación ideológica que articula el programa político de un determinado sector social (clases, fracciones, grupos sociales, etc.) con las estrategias y acciones que impulsan desde el Estado, a través de las políticas educativas, tanto en su formulación discursiva (fines, objetivos, prioridades, etc.), como en su ejecución (estrategias de acción, medios, instrumentos, etc.). El análisis de las reformas educativas requiere distinguir, metodológicamente, al menos tres ámbitos: a) el de la teoría o propuestas de los "expertos", b) el de la legalidad en todas sus manifestaciones, y c) el de las prácticas. Aunque no siempre estas tres dimensiones coinciden, lo cierto es que están articuladas indisolublemente por la racionalidad ideológica a partir de la cual se formulan y se implementan. Según nuestra visión del análisis de la política educativa, el acercamiento hermenéutico crítico es indispensable para una comprensión concreta (en el sentido dialéctico) de las propuestas de reforma o innovaciones educativas a partir de la relación del descubrimiento de su teleología 
concreta, la esencia, el verdadero trasfondo de la intencionalidad simbólica de la reforma e innovación educativas.

Los modelos teóricos en que se basan las reformas educativas se sustentan en determinadas teorías sociales generales; por consiguiente, dan cuenta del funcionamiento global de la sociedad, sus cambios y del papel de la educación en esos cambios. Dichos modelos, como apunta Pablo Latapí "permiten 'encuadrar' (de alli el nombre de marco teórico) la apreciación de las relaciones entre educación y sociedad" (1984, p. 20).

En el ámbito metodológico de la crítica a las políticas educativas, es preciso, en consecuencia, desvelar y explicitar el marco teórico-social en que se sustenta la política educativa como objeto de estudio. Esto es, realizar un análisis interpretativo de sus "modelos teórico-sociales por cuanto constituyen representaciones de la realidad que tienen la pretensión de ser congruentes internamente y de estar basadas en hipótesis en estadios diversos de comprobación" (1984, p. 20) ${ }^{9}$. Los objetivos y/o propósitos de una reforma educativa y su adecuación a sus efectos y consecuencias se deben distinguir entre los propósitos explícitos y los no dichos o implícitos, a veces incluso negados, como lo advierte Viñao (2002).

\section{Dimensión epistemológico-social de las reformas educativas: la contribuición de Thomas W. Popkewitz}

Una comprensión integral y concreta (en el sentido dialéctico del término) de las reformas educativas sólo es posible si se realiza desde una aproximación crítica, que tome en cuenta la dimensión estructural que condiciona su surgimiento y desarrollo sociohistórico. El estudio del significado histórico sociopolítico de las reformas educativas está centrado en responder en qué consiste, el conocimiento de sus significados cambiantes en el tiempo y en dar cuenta de cómo surgen esos significados. Popkewitz (1997) ha brindado un excelente aporte al situar su análisis de las reformas educativas en el campo social de la escolarización y su ecología. Este último aspecto permite determinar los campos en que se produce la reforma, y permite analizar algunos aspectos referentes a los factores que intervienen en las diferentes relaciones entre la enseñanza y la sociedad a través del tiempo. Su tesis central establece que la mejor manera de entender la reforma educativa es considerarla como parte de

9 Pablo Latapí (1984, pp. 19-30) propone cuatro modelos teórico-sociológicos: modernización pedagógica, reformismo social, radicalismo e incrementalismo. 
un proceso de regulación social, en tanto que permite resaltar la importancia de los elementos activos del poder en la producción y disciplina sociales de las capacidades de los individuos.

En consecuencia, su análisis no enfatiza los mecanismos globales de control; antes bien, su preocupación se orienta a explorar las formas en que la reforma educativa está relacionada con las distintas dimensiones propias de los asuntos sociales: desde la organización de las instituciones hasta la autodisciplina, así como la organización de la percepción y la experiencia a través de la cual actúan los sujetos sociales. Se opta por el concepto de regulación, en lugar del concepto de control social, predominante en la sociología de la educación de la década de los setenta. Sin dejar de reconocer dimensiones globales como el capitalismo y la industrialización, su interés está focalizado, más bien, en las formas particulares y los ámbitos a través de los cuales el poder afecta a las relaciones sociales y las produce. Su objeto principal de análisis, por lo tanto, es de qué manera el poder limita y reprime las prácticas sociales.

De manera particular, la preocupación de Popkewitz (1997) es la relación del poder con el saber, pero no cualquier tipo de saber, sino aquel que nos permite expresar deseos personales, voluntades, necesidades corporales e intereses cognitivos. Esta, en esencia, constituye su peculiar contribución al análisis crítico del currículo.

Siendo que su interés es conocer la dialéctica entre el poder y el saber y sus manifestaciones en la enseñanza, realiza su abordaje metodológico recurriendo a las teorías del discurso, con la intención de considerar las reglas y normas de los textos en cuanto prácticas sociales; toma en consideración las condiciones históricas, prácticas institucionales y epistemológicas, más que sobre el habla y los textos en sí mismos. De las consideraciones expuestas, se desprende que la aproximación hermenéutica crítica al estudio de las reformas escolares contemporáneas, según Popkewitz, implica una construcción histórica de las relaciones entre el saber y el poder.

La construcción de visión que asume parte de una interpretación desde el papel que le asigna este autor a la epistemología social: un modo de vincular los cambios estructurales al pensamiento o las ideas educativas en sus diversas formas discursivas y a las reformas como prácticas sociales.

Adquieren esa naturaleza en virtud de que son partes integrantes de una totalidad estructurada, en la cual adquieren su concreción a partir de su carácter genético-dinámico. Su comprensión e interpretación requieren, desde 
el punto de vista dialéctico de la práctica investigativa crítica, conocer sus causas, su génesis social en su dimensión temporal, y su realidad actual en el conjunto más amplio e inmediato de sus determinaciones estructurales. Es por esto que las estructuras adquieren una singular importancia en una doble dimensión: i) se erigen en las dimensiones centrales o categoriales del análisis de la sociedad global y sus principales manifestaciones de la vida colectiva (económica, social, política y cultural); y, por consiguiente, ii) se constituyen en el marco de referencia analítico-crítico de los aspectos fundamentales de la escolarización: los marcos simbólicos de representación, como elementos estructuradores, que corresponden a nociones o referentes que se retraducen en estructuras pensadas que caracterizan las acciones, las prácticas y el pensamiento.

Este proceso incluye aquellos conceptos que en su connotación esconden sus orígenes y sus intenciones en un determinado cuerpo teórico. De igual manera, la realidad educativa como objeto de estudio, en su dimensión global o en algunas de sus expresiones particulares, exige una aproximación hermenéutica crítica que considere su articulación a las distintas dimensiones estructurales del sistema social en su conjunto, desde una visión dialéctica. En efecto, siguiendo a Popkewitz (1997, p. 34), la dimensión estructural de los fenómenos educativos en su condición de ser una de las "formas sociales heredadas de las condiciones sociales históricas" necesita de precisar y analizar críticamente el "conjunto de supuestos o principios mediante los cuales se definen los hechos sociales o se expresa su oposición", así como "los limites cambiantes y los puntos de interacción entre instituciones y sistemas sociales (que) definen las pautas estructurales", este es el análisis crítico de las pautas de relaciones de las instituciones históricamente constituidas.

El cambio educativo refiere todo un conjunto complejo de fenómenos, dimensiones y problemas, y eso mismo exige una necesaria complementación de enfoques y perspectivas para comprenderlo y promoverlo. En el ámbito de los cambios educativos, la aplicación de este punto de vista debe considerar que el conjunto de relaciones que los configuran no son lineales; antes bien, representan procesos estructurales de ruptura con el pasado o de esfuerzos de continuidad históricamente situados. Esta aproximación crítica coloca el currículo como un elemento integrador y estructurador de las relaciones sociales. A este respecto, Popkewitz explica que el concepto de currículo expresa la orientación de las relaciones estructuradas que configuran los hechos de la escolarización, en virtud de que engloba diversos conjuntos de relaciones 
sociales estructurales por medio de las pautas de comunicación en las que se basa (1997, p. 35).

Las aportaciones de Popkewitz brindan una sugerente posibilidad para una aproximación crítica al análisis del currículo en su diseño y como elemento vertebrador de los procesos de aprendizaje, en su doble dimensión de práctica institucional y social. Veamos algunas que a nuestro juicio son fundamentales:

1. Al establecer, desde una perspectiva histórica, las interacciones dialécticas entre el conocimiento escolar y las prácticas sociales e institucionales en las formulaciones curriculares y sus prácticas, contribuye a superar la aparente neutralidad y ahistoricidad con que se asumen los enfoques curriculares, y los contenidos sistematizados en los planes y programas de estudio.(Incluyendo las mediaciones pedagógicas, didácticas y evaluativas.) En particular, nos parece que contribuiría a superar la visiór y las prácticas de diseño curricular tan constreñidas en una práctica instrumental de corte tecnocrático y psicologista de escasa o carente de una reflexión epistemológica social.

2. Permite situar el desarrollo curricular como parte integrante de un proceso más amplio de cambio educativo (por ejemplo las reformas y las innovaciones) enriqueciendo la práctica docente y la enseñanza de los y las estudiantes como una práctica consciente y crítica.

3. Con la introducción del análisis epistemológico social de las formulaciones curriculares (planes, programas de materias, criterios de selección de contenidos, perspectivas de análisis histórico-pedagógico, etc.) trasciende la racionalidad tecnicista e instrumental con que se diseñan y analizan dichas formulaciones (tanto teórica como metodológicamente).

4. Posibilita una compresión integral del currículo que toma en consideración las intenciones políticas desde el más alto nivel, las relaciones institucionales, las mediaciones pedagógicas, las prácticas profesionales y científicas definidas, que a su vez se definen en los conjuntos de pautas culturales que enmarcan los currícula y que facilitan estrategias para la definición del ejercicio docente y de la formación de profesores.

5. La relación saber/poder por la amplitud representa una invaluable posibilidad para el trabajo interdisciplinario y transdisciplinario en la investigación e innovación curricular que abarque aspectos centrales de la nueva temática en boga relativos a los problemas multiculturales, 
género, aplicaciones pedagógicas de las tecnologías de la comunicación y de la información, etc.

\section{Las funciones educativas como referentes socioestructurales de las reformas e innovaciones educativas: la visión de $M$. W. Apple}

La naturaleza de la política educativa, particularmente la orientación sociopolítica y económica de las reformas está articulada al conjunto del sistema educativo y sus instituciones educativas y, por ende, tiene una incidencia fundamental en las prácticas educativas concretas. El correlato concreto de las reformas educativas y sus prácticas definen esa vinculación a partir de tres grandes ámbitos de acción que, según Apple (2000), especifican las funciones del sistema educativo: la acumulación, la legitimación y la producción. Como funciones orientadas a procesos estructurantes del sistema social, están íntimamente relacionadas entre sí -aunque posean amplios márgenes de autonomía- y configuran las dimensiones social, ideológica y económica del aparato educativo. En consecuencia, si se pretende establecer la naturaleza y orientación de las reformas educativas y sus vinculaciones concretas (en un sentido dialéctico) a los cambios socioestructurales, es preciso determinar el carácter que asume la reforma en función de estos tres ámbitos de acción:

1. Acumulación de capital. El sistema educativo contribuye a recrear una economía generadora de desigualdades, por medio de una clasificación y selección de estudiantes con base en el "talento" (Apple, 2000, p. 14). Es decir, las capacidades y méritos se redistribuyen teniendo como referente los requerimientos del mercado laboral, definido con arreglo a una estructura ocupacional, que a su vez responde a un determinado modelo económico que, históricamente, se define en función de la lógica de acumulación de capital. Aquí es interesante poner de relieve que, la base teórica de las formulaciones económicas en esta materia incluye una justificación de naturaleza sociológica, que sirve de fundamento justificativo y racionalizador de los criterios epistemológicos a partir de los cuales se establece la clasificación y la selección de los alumnos. Este aspecto ha sido el tema central del debate sobre la relación educación-empleo desde las corrientes teóricas derivadas del estructural-funcionalismo, el funcionalismo, la teoría funcionalista de la estratificación social o la teoría del capital humano, que han dado origen al funcionalismo tecnoeconómico (Gil Villa, 1994). 
El sustrato epistemológico de estas corrientes teóricas tiene como elemento central la conformación o reproducción de una fuerza laboral organizada a partir de la integración de los estudiantes a un mercado común de antecedentes y a un sistema de segregación. Para ello, esa ordenación jerárquica obedece a un criterio basado en las formas culturales de los grupos dominantes. Así, la clasificación jerárquica del conjunto de valores, normas, hábitos, usos, costumbres, habilidades, conocimientos, destrezas, etc., depende de su pertenencia étnica, sexo y origen social. La contribución del sistema educativo a las demandas del sistema económico consiste en disponer de un "ejército laboral" con cierto grado de socialización y estratificado de conformidad con la estructura ocupacional requerida a los intereses de producción económica. Apple (2000) llama la atención de que no se trata de asumir una posición reduccionista que supedite la relación educación-economía a un modelo simplista y mecanicista de base/superestructura, en el cual los únicos factores que intervienen en esa relación sean las necesidades de la división del trabajo o al desarrollo de las fuerzas productivas. "Sin embargo, evitar tal reduccionismo no significa que podamos ignorar los vínculos reales entre la economía y la actividad de selección y clasificación de la educación" (Apple, 2000, p. 115).

2. Legitimación. Los centros educativos, como elementos constitutivos del sistema educativo y agentes de legitimación, forman parte de una "compleja estructura mediante la cual se legitiman grupos sociales y se recrean, mantienen y construyen continuamente ideologías sociales y culturales" constituyéndose en "agentes de legitimación" (p. 115). La lógica que subyace en la legitimación consiste en no mostrar la realidad social tal como es, sino en ocultar, a través de representaciones deformadas, los intereses de los protagonistas de la lucha ideológica. El mecanismo de la legitimación estriba en que la sociedad no se considere por las representaciones conforme a la realidad de la división social del trabajo y su consecuente la lucha social y política. De aquí que el fin que se persigue es un ocultamiento de los intereses de las clases dirigentes para legitimar el poder constituido (Levi, 1982). En esta perspectiva, es que consideramos que el sistema conceptual gramsciano contribuye para comprender, en forma integral y alejadas de todo reduccionismo economicista, las dimensiones política, ideológica y cultural de la educación en las formaciones sociales capitalistas (de cualquier signo doctrinal político) que están articuladas a la dimensión de la estructura económica. 
Según Apple (2000, pp. 115-116), el sistema educativo contribuye en esta lógica a describirse a sí mismo y a la sociedad como instancias meritocráticas y que se dirigen hacia la búsqueda de la justicia social y económica. En consecuencia, el marco de representaciones ideológicas reproduce la creencia social de que la institucionalidad del régimen político tiene intereses genuinos por la población sin distingos de ninguna índole. Sin embargo, la muy divulgada visión de que las sociedades de capitalismo avanzado poseen una alta dosis de pluralismo ha sido desmentida por investigaciones en las políticas sociales y antiinflacionarias. Apple observa, de la misma forma, que el papel de la escuela en el proceso de legitimación no se circunscribe a fomentar la imagen de que el sistema socioeconómico aparezca como natural o justo, o a marcar los límites entre grupos. Como instituciones políticas que son, las escuelas integran el sistema institucional, es decir, el régimen político de la sociedad y del Estado deben legitimarse a sí mismos. Esto significa, que no sólo la economía, sino el sistema educativo, la burocracia estatal y el gobiemo tienen sus propias necesidades de legitimación y, por consiguiente, la condición de posibilidad de su estabilidad es el consenso entre los gobernados. Sin embargo, aclara que la necesidad de la legitimación política no siempre está en consonancia con las demandas del sistema económico. Y es claro que esto sea así, pues no existe una relación bis a bis entre la esfera política de la sociedad con la esfera económica.

3. Producción de conocimiento. El aparato escolar está concebido como un conjunto de agentes para producir conocimiento. Este proceso es también importante para comprender el papel y el funcionamiento general del sistema escolar y su articulación orgánica y funcional con el sistema económico capitalista, en cuanto a la producción del conocimiento técnico/administrativo requerido en ámbitos como el proceso productivo, la distribución de mercancías o servicios y el consumo, en aspectos tales como: la expansión de los mercados, aplicaciones militares, control y la división de trabajo, innovaciones comunicativas y técnicas dirigidas a aumentar o mantener la participación en un mercado específico o para aumentar las ganancias; pero, además, para mantener el control social (Apple, 2000). En todos los niveles de su estructura, el sistema educativo contribuye en la generación de conocimiento, su enseñanza y su difusión. Los costos de investigación y desarrollo son socializados de manera que todos los contribuyentes sufragan su financiamiento, aliviando las cargas del capital. Tanto la producción que se realiza en industrias de base científica como cultural hacen accesibles estos tipos de 
conocimientos y el entrenamiento a distintos niveles de la fuerza de trabajo para su aplicación.

De esta manera, la formación profesional, las universidades y centros superiores de investigación y formación tecnológica brindan una decidida prestación a través de las innovaciones y capacitación de alto nivel, al desarrollo de las fuerzas productivas y, por ende, a la lógica del proceso de acumulación de capital. La elevación de la composición técnica y orgánica de capital no podría tener lugar sin ese insumo. Otro tipo de conocimiento básico es el conocimiento técnico aplicado en el ámbito organizacional y administrativo a la producción industrial, en el campo gerencial y de gestión empresarial. Este se ha constituido en un elemento básico para la expansión de la producción industrial en su sentido más amplio.

La introducción de la racionalidad instrumental, con el propósito de mejorar los métodos y sistemas de producción a las unidades productivas, ha sido, ciertamente, un factor indispensable para el surgimiento y expansión de la producción en los países capitalistas avanzados.

Al mismo tiempo, el conocimiento técnico-administrativo reduce las prácticas educativas a un entrenamiento para que los estudiantes, futuros ciudadanos, asimilen y luego socialicen en las prácticas del conocimiento que las clases dirigentes consideran legítimo para la producción y lógica mercantil; y en un sentido más general, coadyuva en la implantación de la cultura hegemónica de la democracia liberal. Es este, sin duda, un aspecto esencial en la reproducción de la legitimidad social y política del sistema capitalista, de su régimen institucional-político y de su estructura social.

En el ámbito del sistema educativo, la producción de conocimiento técnico expresa la aplicación de la racionalidad instrumental aplicada a la organización, administración, métodos didácticos y administración curricular, mediatizando los procesos de enseñanza y aprendizaje a una visión reduccionista y conductista del conocimiento y de la realidad.

En la práctica, el resultado final es una "instrumentalización" del estudiante, que circunscribirá su práctica laboral a una mecánica y exclusiva aplicación de destrezas técnicas aplicadas a la producción.

Apple pone de relieve las implicaciones socioculturales que este tipo de prácticas escolares posee, cuando se reduce el conocimiento a conductas atomistas, reduciendo, al mismo tiempo, la esfera cultural a la aplicación de reglas y procedimientos técnicos. Los enfoques curriculares desde el punto de vista del conocimiento técnico-administrativo desempeñan un papel crucial en la conformación de una mentalidad y una actitud acrítica, 
sustrayendo de la formación la dimensión ética y moral de la política, la cultura y la economía. En realidad, lo que ocurre es una cosificación, un vaciamiento de la dimensión integral humana y social del aprendizaje para el trabajo, y con ello del trabajador, el cual queda reducido a un simple valor deshumanizado al concebirse como un componente más del engranaje del proceso productivo (2000, p. 117).

Es importante poner de relieve, empero, que las funciones que Apple identifica como básicas en las políticas educativas (la acumulación, la legitimación y la producción) pueden resultar a menudo contradictorias, e incluso puede actuar alguna en detrimento de las otras. Apple recurre al ejemplo de los contextos de crisis fiscal, en los cuales la industria requiere de menos trabajadores de alta calificación y altos ingresos, que trae aparejada la crisis del sistema educativo y del gobiemo. Aunque este autor no hace una referencia específica a la crisis de legitimación, en realidad a eso se está refiriendo cuando afirma que "en este proceso, otros sectores de la población pueden perder confianza en la autoridad y legitimidad del gobiemo, si perciben el ajuste como generador de desigualdad y como una limitación de los caminos que necesitan para progresar (p. 118). Es decir, hay situaciones en que las expectativas personales en la población están en franca contradicción con las medidas del gobiemo de realizar ajustes económicos que modifican las políticas educativas. Esto decididamente afecta las funciones del aparato escolar.

La política educacional queda atrapada en una situación contradictoria. Debe colaborar en la recreación de un orden económico e ideológico relativamente estrecho, mientras que mantiene su legitimidad ante los ojos de los otros. Así, la necesidad de consenso del Estado está a veces reñida con las presiones que le han impuesto las condiciones económicas cambiantes (Apple, 2000, p. 119).

A nuestro parecer, esta problemática en el contexto de la crisis de legitimación, cuando existe déficit de consenso, se explica mejor por el enfoque tanto de Habermas como de Offe de las teorías de la crisis de legitimación y de las crisis sistémicas desplazadas, desde el sistema económico hasta el sistema cultural, sobre la cual ya nos hemos referido en otra oportunidad ${ }^{10}$. Apple se refiere también al potencial contrahegemónico que por su relativa autonomía poseen los centros escolares de desarrollar prácticas culturales alternativas y/o

10 La utilización de la teoría de la crisis de legitimación y de la teoria de la crisis sistémica desplazada de Habermas y Offe como marcos analítico-críticos de la reforma educativa, la hemos examinado en el precitado artículo "Política curricular, crisis de legitimación y hegemonia neoliberal. Una visión hermenéutica desde la sociología de la educación crítica” (véase cita $\mathrm{N}^{\circ} 6$ ). 
de oposición. No deja de sorprendemos que este autor, que recurre a algunas dimensiones teórico-analíticas de la teoría crítica en su análisis, no tome en consideración aspectos como los teoremas sobre la crisis de legitimación. 


\section{REFERENCIAS}

Angulo, R. F. y Blanco, M. N. (1994). Innovación, cambio y reforma: Algunas ideas para analizar lo que está ocurriendo, teoría y desarrollo del currículo. Málaga: ALJIVE.

Apple, Michel W. (1997). Educación y poder. Madrid: PAIDÓS.

Apple, Michel W. (2000). Teoría crítica y educación. Madrid.

Castells, M. (2002). La galaxia Internet. Barcelona: DEBOLSILLO.

Chalmers, A. (2000). ¿Qué es esa cosa llamada ciencia? Madrid: SIGLO XXI.

Colom, A. y Domínguez, E. (1997). Introducción a la política de la educación. Barcelona: ARIEL.

Eagleton, Terry (1997). Ideología una introducción. Barcelona: PAIDÓS.

Fernández, S. y Pérez, M. (1995). Politica educativa y sociedad. Valencia: AU LLIBRES.

Fische, L. (1982). Tecnocracia. Diccionario de politica. Madrid: TECNOS.

García Femández, M. (1998). Diseño, desarrollo e innovación del curriculum. Córdoba: Servicio de Publicaciones, Universidad de Córdoba.

Gil V., Fernando (1994). Teoría sociológica de la educación. Salamanca: AMARÚ EDICIONES.

González González, M. y Escudero Muñoz, J. (1987). Innovación educativa: Teoria y procesos de desarrollo. Barcelona: EDITORIAL HUMANITAS.

Habermas, J. (2001). Ciencia y técnica como ideologia. Madrid: TECNOS.

Latapí, P. (1984). Análisis de un sexenio de educación en México, 1970-1976.

Laval (2004). La escuela no es una empresa. Barcelona: PAIDÓS.

Levi, L., Bobbio, Norberto y Matteucci, N. (1982). Legitimidad. Diccionario de politica. Madrid: TECNOS.

Mandel, Emest (1986). Las ondas largas del desarrollo capitalista. Madrid: SIGLO XXI.

Marcuse, H. (1972). El hombre unidimensional. Barcelona: SEIX BARRAL.

Miranda, G. (2006). Hacia una visión hermenéutico critica de la política educativa, pp. 111-112.

Miranda, G. "Política curricular, crisis de legitimación y hegemonía neoliberal". En Revista de Ciencias Sociales. № 112-113 (no publicada). San José: Editorial Universidad de Costa Rica.

Moral, S. y Raymond, H. (1986). La acumulación del capital y sus crisis. Madrid, AKAL.

Ordóñez P., J. (2004). Introducción a la pedagogía. San José: EUNED.

Pérez G., Á. (1999). La cultura escolar en la sociedad neoliberal. Madrid: MORATA. 
Popkewitz, T. S. (1997). Sociologia Politica de las Reformas Educativas. Madrid. MORATA.

Postic, Marcel (1982). La relación educativa. Madrid: NANCEA.

Requeijo, J. (2000). Estructura económica mundial. Madrid: MC GRAW-HILL.

Rudduck, J., Angulo, R., Félix, J. y Blanco, N. (1994). Reflexiones sobre el problema del cambio en las escuelas. En Teoria y Desarrollo del Curriculo. Málaga: ALJVE.

Viñao, A. (2002). Sistemas educativos, culturas escolares y reformas. Madrid: MORATA. 